\title{
Multiple positive solutions of nonlinear third-order boundary value problems with integral boundary conditions on time scales
}

\author{
Yongkun Li* and Lingyan Wang
}

*Correspondence: yklie@ynu.edu.cn Department of Mathematics, Yunnan University, Kunming, Yunnan 650091, People's Republic of China

\begin{abstract}
In this paper, we consider a class of nonlinear third-order boundary value problems with integral boundary conditions on time scales. By applying a generalization of the Leggett-Williams fixed point theorem, we establish the existence of at least three positive solutions. The discussed problem involves both an increasing and positive homomorphism, which generalizes the $p$-Laplacian operator. As an application, we give an example to illustrate our results.
\end{abstract}

Keywords: time scales; fixed point theorem; integral boundary conditions; multiple positive solutions; cone

\section{Introduction}

In recent years, much attention has been paid to boundary value problems with integral boundary conditions due to their various applications in chemical engineering, thermoelasticity, population dynamics, heat conduction, chemical engineering underground water flow, thermo-elasticity, and plasma physics. Many results for the boundary value problems with integral boundary conditions have been reported in the literature (see [1-5] and references therein). For example, in [5], Guo et al. considered the following boundary value problem:

$$
\left\{\begin{array}{l}
u^{\prime \prime \prime}(t)+f\left(t, u^{\prime}(t), u^{\prime \prime}(t)\right)=0, \quad t \in(0,1), \\
u(0)=0, \quad u^{\prime \prime}(0)=0, \quad u(1)=\int_{0}^{1} g(t) u(t) d t
\end{array}\right.
$$

where $f:[0,1] \times \mathbf{R}^{+} \times \mathbf{R}^{-} \rightarrow \mathbf{R}^{+}$is a nonnegative function. By applying the fixed point index theory in a cone and spectral radius of a linear operator, they found that (1) has at least one positive solution.

On the other hand, the study of dynamic equations on time scales, which has been created in order to unify the study of differential and difference equations, is an area of mathematics that has recently received a lot of attention; moreover, many results on this issue have been well documented in the monographs [6-8].

In addition, boundary value problems with integral boundary conditions on time scales represent a very interesting and important class of problems. The study of boundary value problems on time scales has received a lot attention in the literature (see [9-18]). For example, in [17], Li and Zhang studied the second-order $p$-Laplacian dynamic equation on 
time scales,

$$
\left(\varphi_{p}\left(x^{\Delta}(t)\right)\right)^{\nabla}+\lambda f\left(t, x(t), x^{\Delta}(t)\right)=0, \quad t \in(0, T)_{\mathbb{T}},
$$

with integral boundary condition

$$
x^{\Delta}(0)=0, \quad \alpha x(T)-\beta x(0)=\int_{T}^{0} g(s) x(s) \nabla s .
$$

By using the Legget-Williams fixed point theorem, they obtained some criteria for the existence of at least three positive solutions to problems (2)-(3).

In [18], Han and Kang considered the existence of multiple positive solutions for the following third-order $p$-Laplacian dynamic equation on time scales:

$$
\left\{\begin{array}{l}
\left(\Phi_{p}\left(u^{\Delta \Delta}(t)\right)\right)^{\nabla}+f(t, u(t))=0, \quad t \in[a, b] \\
\alpha u(\rho(a))-\beta u^{\Delta}(\rho(a))=0 \\
\gamma u(b)+\delta u^{\Delta}(b)=0 \\
u^{\Delta \Delta}(\rho(a))=0
\end{array}\right.
$$

By using fixed point theorems in cones, they obtained the existence criteria of at least two positive solutions to problem (4).

However, to the best of our knowledge, there is no paper published on the existence of multiple positive solutions to nonlinear third-order boundary value problems with integral boundary conditions on time scales. This paper attempts to fill this gap in the literature.

Motivated by the above, in this paper, we are concerned with the following third-order boundary value problem with integral boundary conditions on time scale $\mathbb{T}$ :

$$
\left\{\begin{array}{l}
\left(\phi\left(-u^{\Delta \Delta}(t)\right)\right)^{\Delta}+q(t) f\left(t, u(t), u^{\Delta}(t)\right)=0, \quad t \in[0,1]_{\mathbb{T}} \\
a u(0)-b u^{\Delta}(0)=\int_{0}^{1} g_{1}(s, u(s)) \Delta s \\
c u(1)+d u^{\Delta}(1)=\int_{0}^{1} g_{2}(s)(u(s)) \Delta s \\
u^{\Delta \Delta}(1)=0
\end{array}\right.
$$

where $\mathbb{T}$ is a time scale, 0 and 1 are points in $\mathbb{T},[0,1]_{\mathbb{T}}:=[0,1] \cap \mathbb{T}, \phi: \mathbf{R} \rightarrow \mathbf{R}$ is an increasing and positive homeomorphism (see Definition 2.3) with $\phi(0)=0$.

Our main purpose of this paper is to study the existence of multiple positive solutions to (5). Our method of this paper is based on some recent fixed point theorems derived by Bai and Ge (see [19]). As an application, we give an example to illustrate our results.

Throughout this paper, we assume that the following conditions are satisfied:

$\left(C_{1}\right) a, b, c, d \in[0,+\infty)$ with $\rho:=a c+a d+b c>0$,

$\left(C_{2}\right) f \in C\left([0,1]_{\mathbb{T}} \times \mathbf{R}^{+} \times \mathbf{R}^{+}, \mathbf{R}^{+}\right)$with $f(t, 0,0) \neq 0$ for all $t \in[0,1]_{\mathbb{T}}$,

$\left(C_{3}\right) g_{1} \in C\left([0,1]_{\mathbb{T}} \times \mathbf{R}^{+}, \mathbf{R}^{+}\right), g_{2} \in C\left([0,1]_{\mathbb{T}}, \mathbf{R}^{+}\right)$and $q \in C\left([0,1]_{\mathbb{T}}, \mathbf{R}^{+}\right)$.

$\left(\mathrm{C}_{4}\right) \rho-\int_{0}^{1} g_{2}(s)(b+a s) \Delta s>0$.

\section{Preliminaries and statements}

In this section, we provide some background materials from theory of cones in Banach spaces. The following definitions can be found in the book by Deimling [20] as well as in the book by Guo and Lakshmikantham [21]. 
Definition 2.1 Let $\mathbb{E}$ be a real Banach space. A nonempty closed convex set $P \subset \mathbb{E}$ is called a cone if it satisfies the following two conditions:

(i) $x \in P, \lambda \geq 0$ implies $\lambda x \in P$;

(ii) $x \in P,-x \in P$ implies $x=0$.

Every cone $P \subset \mathbb{E}$ induces an ordering in $\mathbb{E}$ given by $x \leq y$ if and only if $y-x \in P$.

Definition 2.2 A map $\psi$ is said to be a nonnegative continuous concave functional on a cone $P$ of a real Banach space $\mathbb{E}$ if $\psi: P \rightarrow[0, \infty)$ is continuous and

$$
\psi(t x+(1-t) y) \geq t \psi(x)+(1-t) \psi(y)
$$

for all $x, y \in P$ and $t \in[0,1]$. Similarly, we say that a map $\alpha$ is a nonnegative continuous convex functional on a cone $P$ of a real Banach space $\mathbb{E}$ if $\alpha: P \rightarrow[0, \infty)$ is continuous and

$$
\alpha(t x+(1-t) y) \leq t \alpha(x)+(1-t) \alpha(y)
$$

for all $x, y \in P$ and $t \in[0,1]$.

Definition 2.3 A projection $\phi: \mathbf{R} \rightarrow \mathbf{R}$ is called an increasing and positive homomorphism if the following conditions are satisfied:

(i) If $x \leq y$, then $\phi(x) \leq \phi(y)$ for all $x, y \in \mathbf{R}$;

(ii) $\phi$ is a continuous bijection, and its inverse mapping is also continuous;

(iii) $\phi(x y)=\phi(x) \phi(y)$ for all $x, y \in \mathbf{R}^{+}$, where $\mathbf{R}^{+}=[0,+\infty)$.

Let $\psi$ be a nonnegative continuous concave functional on $P$, and $\alpha$ and $\beta$ be nonnegative continuous convex functionals on $P$. For nonnegative real numbers $r, a$, and $l$, we define the following convex sets:

$$
\begin{aligned}
& P(\alpha, r ; \beta, l)=\{u \in P: \alpha(u)<r, \beta(u)<l\}, \\
& \bar{P}(\alpha, r ; \beta, l)=\{u \in P: \alpha(u) \leq r, \beta(u) \leq l\}, \\
& P(\alpha, r ; \beta, l ; \psi, a)=\{u \in P: \alpha(u)<r, \beta(u)<l, \psi(u)>a\}, \\
& \bar{P}(\alpha, r ; \beta, l ; \psi, a)=\{u \in P: \alpha(u) \leq r, \beta(u) \leq l, \psi(u) \geq a\} .
\end{aligned}
$$

To prove our main results, we need the following fixed point theorem, which comes from Bai and Ge in [19].

Lemma 2.1 [19] Let $P$ be a cone in real Banach space $\mathbb{E}$. Assume that constants $r_{1}, b, d, r_{2}$, $l_{1}$, and $l_{2}$ satisfy $0<r_{1}<b<d \leq r_{2}$ and $0<l_{1} \leq l_{2}$. If there exist two nonnegative continuous convex functionals $\alpha$ and $\beta$ on $P$ and a nonnegative continuous concave functional $\psi$ on $P$ such that:

$\left(\mathrm{A}_{1}\right)$ there exists $M>0$ such that $\|u\| \leq M \max \{\alpha(u), \beta(u)\}$ for all $u \in P$;

$\left(\mathrm{A}_{2}\right) P(\alpha, r ; \beta, l) \neq \emptyset$ for any $r>0$ and $l>0$;

$\left(\mathrm{A}_{3}\right) \psi(u) \leq \alpha(u)$ for all $u \in \bar{P}\left(\alpha, r_{2} ; \beta, l_{2}\right)$;

and if $F: \bar{P}\left(\alpha, r_{2} ; \beta, l_{2}\right) \rightarrow \bar{P}\left(\alpha, r_{2} ; \beta, l_{2}\right)$ is a completely continuous operator which satisfies

$\left(\mathrm{B}_{1}\right)\left\{u \in \bar{P}\left(\alpha, d ; \beta, l_{2} ; \psi, b: \psi(u)>b\right)\right\} \neq \emptyset, \psi(F u)>b$ for $u \in \bar{P}\left(\alpha, d ; \beta, l_{2} ; \psi, b\right)$; 
$\left(\mathrm{B}_{2}\right) \alpha(F u)<r_{1}, \beta(F u)<l_{1}$ for $u \in \bar{P}\left(\alpha, r_{1} ; \beta, l_{1}\right)$;

$\left(\mathrm{B}_{3}\right) \psi(F u)>$ bor $u \in \bar{P}\left(\alpha, r_{2} ; \beta, l_{2} ; \psi, b\right)$ with $\alpha(F u)>d$,

then $F$ has at least three different fixed points $u_{1}, u_{2}$, and $u_{3}$ in $\bar{P}\left(\alpha, r_{2} ; \beta, l_{2}\right)$ with

$$
\begin{aligned}
& u_{1} \in P\left(\alpha, r_{1} ; \beta, l_{1}\right), \quad u_{2} \in\left\{\bar{P}\left(\alpha, r_{2} ; \beta, l_{2} ; \psi, b: \psi(u)>b\right)\right\} \\
& u_{3} \in \bar{P}\left(\alpha, r_{2} ; \beta, l_{2}\right) \backslash\left(\bar{P}\left(\alpha, r_{2} ; \beta, l_{2} ; \psi, b\right) \cup \bar{P}\left(\alpha, r_{1} ; \beta, l_{1}\right)\right) .
\end{aligned}
$$

Let $\mathbb{E}=C^{\Delta}[0,1]=\left\{u\right.$ and $u^{\Delta}$ are continuous on $\left.[0,1]_{\mathbb{T}}\right\}$. Then $\mathbb{E}$ is a Banach space with respect to the norm

$$
\|u\|=\max \left\{\max _{t \in[0,1]_{\mathbb{T}}}|u(t)|, \max _{t \in[0,1]_{\mathbb{T}}}\left|u^{\Delta}(t)\right|\right\} .
$$

Define

$$
P=\left\{u \in \mathbb{E}: u(t) \geq 0, u^{\Delta}(t) \geq 0, u(t) \text { is concave on }[0,1]_{\mathbb{T}}\right\} .
$$

Clearly, $P$ is a cone.

Lemma 2.2 Assume that $\left(\mathrm{C}_{1}\right)-\left(\mathrm{C}_{3}\right)$, and $\rho-\int_{0}^{1} g_{2}(s)(b+a s) \Delta s \neq 0$ hold. Then $u \in \mathbb{E}$ is a solution of the boundary value problem (5) if and only if $u(t)$ is a solution of the following integral equation:

$$
u(t)=\int_{0}^{1} G(t, s) \phi^{-1}\left(\int_{s}^{1} q(\tau) f\left(\tau, u(\tau), u^{\Delta}(\tau)\right) \Delta \tau\right) \Delta s+(d+c(1-t)) \Phi_{f}+(b+a t) \Psi_{f},
$$

where

$$
\begin{aligned}
G(t, s)= & \frac{1}{\rho} \begin{cases}(b+a \sigma(s))(d+c(1-t)), & 0 \leq \sigma(s) \leq t \leq 1, \\
(b+a t)(d+c(1-\sigma(s))), & 0 \leq t \leq s \leq 1,\end{cases} \\
\Phi_{f}:= & \frac{1}{\rho} \int_{0}^{1} g_{1}(s, u(s)) \Delta s, \\
\Psi_{f}:= & {\left[\rho-\int_{0}^{1} g_{2}(s)(b+a s) \Delta s\right]^{-1}\left[\int_{0}^{1} g_{2}(s) \Gamma_{f}(s) \Delta s\right.} \\
& \left.+\frac{1}{\rho}\left(\int_{0}^{1} g_{2}(s)(d+c(1-s)) \Delta s\right) \int_{0}^{1} g_{1}(s, u(s)) \Delta s\right], \\
\Gamma_{f}(t): & \int_{0}^{1} G(t, s) \phi^{-1}\left(\int_{s}^{1} q(\tau) f\left(\tau, u(\tau), u^{\Delta}(\tau)\right) \Delta \tau\right) \Delta s .
\end{aligned}
$$

Proof Let

$$
\begin{aligned}
u(t)= & \int_{0}^{t} \frac{1}{\rho}(b+a \sigma(s))(d+c(1-t)) \phi^{-1}\left(\int_{s}^{1} q(\tau) f\left(\tau, u(\tau), u^{\Delta}(\tau)\right) \Delta \tau\right) \Delta s \\
& +\int_{t}^{1} \frac{1}{\rho}(b+a t)(d+c(1-\sigma(s))) \phi^{-1}\left(\int_{s}^{1} q(\tau) f\left(\tau, u(\tau), u^{\Delta}(\tau)\right) \Delta \tau\right) \Delta s \\
& +(d+c(1-t)) \Phi_{f}+(b+a t) \Psi_{f} .
\end{aligned}
$$


Taking the $\Delta$-derivative of (9), we get

$$
\begin{aligned}
u^{\Delta}(t)= & -\int_{0}^{t} \frac{c}{\rho}(b+a \sigma(s)) \phi^{-1}\left(\int_{s}^{1} q(\tau) f\left(\tau, u(\tau), u^{\Delta}(\tau)\right) \Delta \tau\right) \Delta s \\
& +\int_{t}^{1} \frac{a}{\rho}(d+c(1-\sigma(s))) \phi^{-1}\left(\int_{s}^{1} q(\tau) f\left(\tau, u(\tau), u^{\Delta}(\tau)\right) \Delta \tau\right) \Delta s \\
& -c \Phi_{f}+a \Psi_{f} .
\end{aligned}
$$

It follows that

$$
\begin{aligned}
u^{\Delta \Delta}(t)= & \frac{1}{\rho}(-c(b+a \sigma(t))-a(d+c(1-\sigma(t)))) \\
& \times \phi^{-1}\left(\int_{t}^{1} q(\tau) f\left(\tau, u(\tau), u^{\Delta}(\tau)\right) \Delta \tau\right) \\
= & -\frac{1}{\rho}(a c+a d+b c) \phi^{-1}\left(\int_{t}^{1} q(\tau) f\left(\tau, u(\tau), u^{\Delta}(\tau)\right) \Delta \tau\right) \\
= & -\phi^{-1}\left(\int_{t}^{1} q(\tau) f\left(\tau, u(\tau), u^{\Delta}(\tau)\right) \Delta \tau\right),
\end{aligned}
$$

hence, $u^{\Delta \Delta}(1)=-\phi^{-1}(0)=0$ and

$$
\phi\left(-u^{\Delta \Delta}(t)\right)=\int_{t}^{1} q(\tau) f\left(\tau, u(\tau), u^{\Delta}(\tau)\right) \Delta \tau
$$

so

$$
\left(\phi\left(-u^{\Delta \Delta}(t)\right)\right)^{\Delta}=-q(t) f\left(t, u(t), u^{\Delta}(t)\right),
$$

that is,

$$
\left(\phi\left(-u^{\Delta \Delta}(t)\right)\right)^{\Delta}+q(t) f\left(t, u(t), u^{\Delta}(t)\right)=0
$$

Furthermore, we have

$$
\begin{aligned}
& u(0)=\int_{0}^{1} \frac{b}{\rho}(d+c(1-\sigma(s))) \phi^{-1}\left(\int_{s}^{1} q(\tau) f\left(\tau, u(\tau), u^{\Delta}(\tau)\right) \Delta \tau\right) \Delta s+(d+c) \Phi_{f}+b \Psi_{f} \\
& u^{\Delta}(0)=\int_{0}^{1} \frac{a}{\rho}(d+c(1-\sigma(s))) \phi^{-1}\left(\int_{s}^{1} q(\tau) f\left(\tau, u(\tau), u^{\Delta}(\tau)\right) \Delta \tau\right) \Delta s-c \Phi_{f}+a \Psi_{f}
\end{aligned}
$$

hence

$$
\begin{aligned}
a u(0)-b u^{\Delta}(0) & =\rho \Phi_{f} \\
& =\int_{0}^{1} g_{1}(s, u(s)) \Delta s .
\end{aligned}
$$

Since

$$
u(1)=\int_{0}^{1} \frac{d}{\rho}(b+a \sigma(s)) \phi^{-1}\left(\int_{s}^{1} q(\tau) f\left(\tau, u(\tau), u^{\Delta}(\tau)\right) \Delta \tau\right) \Delta s+d \Phi_{f}+(b+a) \Psi_{f},
$$




$$
u^{\Delta}(1)=-\int_{0}^{1} \frac{c}{\rho}(b+a \sigma(s)) \phi^{-1}\left(\int_{s}^{1} q(\tau) f\left(\tau, u(\tau), u^{\Delta}(\tau)\right) \Delta \tau\right) \Delta s-c \Phi_{f}+a \Psi_{f}
$$

we obtain

$$
\begin{aligned}
c u(1)+d u^{\Delta}(1)= & \rho \Psi_{f} \\
= & \int_{0}^{1} g_{2}(\zeta)\left(\int_{0}^{1} G(\zeta, s) \phi^{-1}\left(\int_{s}^{1} q(\tau) f\left(\tau, u(\tau), u^{\Delta}(\tau)\right) \Delta \tau\right) \Delta s\right. \\
& \left.+(d+c(1-\zeta)) \Phi_{f}+(b+a \zeta) \Psi_{f}\right) \Delta \zeta .
\end{aligned}
$$

From (11) and (12), we find that $\Phi_{f}$ and $\Psi_{f}$ satisfy

$$
\left\{\begin{array}{l}
\Phi_{f}=\frac{1}{\rho} \int_{0}^{1} g_{1}(s, u(s)) \Delta s \\
\quad\left[-\int_{0}^{1} g_{2}(s)(d+c(1-s)) \Delta s\right] \Phi_{f}+\left[\rho-\int_{0}^{1} g_{2}(s)(b+a s) \Delta s\right] \Psi_{f} \\
\quad=\int_{0}^{1} g_{2}(s) \Gamma_{f}(s) \Delta s
\end{array}\right.
$$

which implies that $\Phi_{f}$ and $\Psi_{f}$ are defined by (7) and (8), respectively. The proof is complete.

Define an operator $F: P \rightarrow \mathbb{E}$ by

$$
\begin{aligned}
(F u)(t)= & \int_{0}^{1} G(t, s) \phi^{-1}\left(\int_{s}^{1} q(\tau) f\left(\tau, u(\tau), u^{\Delta}(\tau)\right) \Delta \tau\right) \Delta s \\
& +(d+c(1-t)) \Phi_{f}+(b+a t) \Psi_{f}
\end{aligned}
$$

where $G, \Phi_{f}$, and $\Psi_{f}$ are defined by (6), (7), and (8), respectively.

Lemma 2.3 Assume that $\left(\mathrm{C}_{1}\right)-\left(\mathrm{C}_{4}\right)$ hold. Then for $u \in P$, we have:

(i) $(F u)(t)$ is concave on $[0,1]_{\mathbb{T}}$.

(ii) $(F u)(t) \geq 0$ for $t \in[0,1]_{\mathbb{T}}$.

Proof For $u \in P$ :

(i) By the definition of $F$ and similar to the proof of (10), we have

$$
(F u)^{\Delta \Delta}(t)=-\phi^{-1}\left(\int_{t}^{1} q(\tau) f\left(\tau, u(\tau), u^{\Delta}(\tau)\right) \Delta \tau\right) \leq 0,
$$

so $(F u)^{\Delta}(t)$ is nonincreasing. This implies that $(F u)(t)$ is concave.

(ii) From (6) and (13), we can verify that $(F u)(t) \geq 0$ for $t \in[0,1]_{\mathbb{T}}$.

The proof is complete.

Lemma 2.4 Let $\left(\mathrm{C}_{1}\right)-\left(\mathrm{C}_{4}\right)$ hold. Assume that

$\left(\mathrm{C}_{5}\right) c-\int_{0}^{1} g_{2}(s) \Delta s<0$.

Then $(F u)^{\Delta}(t) \geq 0$ for $u \in P$ and $t \in[0,1]_{\mathbb{T}}$. 
Proof On the contrary, we assume that the inequality $(F u)^{\Delta}(t)<0$ holds for $u \in P$ and $t \in[0,1]_{\mathbb{T}}$. By Lemma 2.3 , we see that $(F u)^{\Delta}(t)$ is nonincreasing on $[0,1]_{\mathbb{T}}$. Hence

$$
(F u)^{\Delta}(1) \leq(F u)^{\Delta}(t), \quad t \in[0,1]_{\mathbb{T}}
$$

Similar to the proof of (12), we easily obtain

$$
c(F u)(1)+d(F u)^{\Delta}(1)=\int_{0}^{1} g_{2}(s)(F u)(s) \Delta s .
$$

Hence

$$
-\frac{c}{d}(F u)(1)+\frac{1}{d} \int_{0}^{1} g_{2}(s)(F u)(s) \Delta s=(F u)^{\Delta}(1) \leq(F u)^{\Delta}(t)<0 .
$$

Clearly, we have

$$
(F u)(1) \int_{0}^{1} g_{2}(s) \Delta s=\int_{0}^{1} g_{2}(s)(F u)(1) \Delta s \leq \int_{0}^{1} g_{2}(s)(F u)(s) \Delta s<c(F u)(1),
$$

that is,

$$
\left(c-\int_{0}^{1} g_{2}(s) \Delta s\right)(F u)(1)>0 .
$$

According to Lemma 2.3, we have $(F u)(1) \geq 0$. So, $c-\int_{0}^{1} g_{2}(s) \Delta s>0$. However, this contradicts condition $\left(\mathrm{C}_{5}\right)$. Consequently, $(F u)^{\Delta}(t) \geq 0$ for $t \in[0,1]_{\mathbb{T}}$. The proof is complete.

Lemma 2.5 Suppose that $\left(\mathrm{C}_{1}\right)-\left(\mathrm{C}_{5}\right)$ hold. Then $F: P \rightarrow P$ is a completely continuous operator.

Proof From Lemmas 2.3 and 2.4 it follows that $F: P \rightarrow P$ is well defined. Next, we show that $F$ is completely continuous. To this end, we assume that $m$ is a positive constant and $u \in \bar{P}_{m}=\{u \in P:\|u\| \leq m\}$. Note that the continuity of $f\left(t, u(t), u^{\Delta}(t)\right)$ and $q(t)$ guarantees that there is an $M>0$ such that $q(t) f\left(t, u(t), u^{\Delta}(t)\right) \leq \phi(M)$ for all $t \in[0,1]_{\mathbb{T}}$. Therefore, according to Lemma 2.4 and Lemma 2.3(i), we have

$$
\begin{aligned}
\max _{t \in[0,1]_{\mathbb{T}}}|(F u)(t)|= & (F u)(1) \\
= & \int_{0}^{1} \frac{d}{\rho}(b+a \sigma(s)) \phi^{-1}\left(\int_{s}^{1} q(\tau) f\left(\tau, u(\tau), u^{\Delta}(\tau)\right) \Delta \tau\right) \Delta s \\
& +d \Phi_{f}+(b+a) \Psi_{f} \\
\leq & \int_{0}^{1} \frac{d}{\rho}(b+a) \phi^{-1}\left(\int_{0}^{1} q(\tau) f\left(\tau, u(\tau), u^{\Delta}(\tau)\right) \Delta \tau\right) \Delta s+d \Phi_{f}+(b+a) \Psi_{f} \\
\leq & \int_{0}^{1} \frac{d}{\rho}(b+a) \phi^{-1}\left(\int_{0}^{1} \phi(M) \Delta \tau\right) \Delta s+d \Phi_{f}+(b+a) \Psi_{f} \\
\leq & \frac{d M}{\rho}(b+a)+d \Phi_{f}+(b+a) \Psi_{f}
\end{aligned}
$$


and

$$
\begin{aligned}
\max _{t \in[0,1]_{\mathbb{T}}}\left|(F u)^{\Delta}(t)\right|= & (F u)^{\Delta}(0) \\
= & \int_{0}^{1} \frac{a}{\rho}(d+c(1-\sigma(s))) \phi^{-1}\left(\int_{s}^{1} q(\tau) f\left(\tau, u(\tau), u^{\Delta}(\tau)\right) \Delta \tau\right) \Delta s \\
& -c \Phi_{f}+a \Psi_{f} \\
\leq & \int_{0}^{1} \frac{a}{\rho}(d+c) \phi^{-1}\left(\int_{0}^{1} q(\tau) f\left(\tau, u(\tau), u^{\Delta}(\tau)\right) \Delta \tau\right) \Delta s+a \Psi_{f} \\
\leq & \int_{0}^{1} \frac{a}{\rho}(d+c) \phi^{-1}\left(\int_{0}^{1} \phi(M) \Delta \tau\right) \Delta s+a \Psi_{f} \\
\leq & \frac{a M}{\rho}(d+c)+a \Psi_{f},
\end{aligned}
$$

which imply that $F \bar{P}_{m}$ is uniformly bounded. In addition, since $F \bar{P}_{m}$ is uniformly bounded, according to the mean value theorem (Theorem 1.14 in [7]), one can easily see that, for $u \in \bar{P}_{m}, t_{1}, t_{2} \in[0,1]_{\mathbb{T}}$,

$$
\left|(F u)\left(t_{2}\right)-(F u)\left(t_{1}\right)\right| \rightarrow 0, \quad \text { as } t_{2}-t_{1} \rightarrow 0 .
$$

From (14), for all $u \in \bar{P}_{m}, t \in[0,1]_{\mathbb{T}}$, it follows that

$$
(F u)^{\Delta}(t)=(F u)^{\Delta}(0)-\int_{0}^{t} \phi^{-1}\left(\int_{s}^{1} q(\tau) f\left(\tau, u(\tau), u^{\Delta}(\tau)\right) \Delta \tau\right) \Delta s .
$$

Hence, for all $u \in \bar{P}_{m}, t_{1}, t_{2} \in[0,1]_{\mathbb{T}}$, we have

$$
\begin{aligned}
\left|(F u)^{\Delta}\left(t_{2}\right)-(F u)^{\Delta}\left(t_{1}\right)\right|= & \mid \int_{0}^{t_{1}} \phi^{-1}\left(\int_{s}^{1} q(\tau) f\left(\tau, u(\tau), u^{\Delta}(\tau)\right) \Delta \tau\right) \Delta s \\
& -\int_{0}^{t_{2}} \phi^{-1}\left(\int_{s}^{1} q(\tau) f\left(\tau, u(\tau), u^{\Delta}(\tau)\right) \Delta \tau\right) \Delta s \mid \\
& =\left|\int_{t_{2}}^{t_{1}} \phi^{-1}\left(\int_{s}^{1} q(\tau) f\left(\tau, u(\tau), u^{\Delta}(\tau)\right) \Delta \tau\right) \Delta s\right| \\
& \leq\left|\int_{t_{2}}^{t_{1}} \phi^{-1}(\phi(M)) \Delta s\right| \\
& =M\left|t_{2}-t_{1}\right|
\end{aligned}
$$

which implies that

$$
\left|(F u)^{\Delta}\left(t_{2}\right)-(F u)^{\Delta}\left(t_{1}\right)\right| \rightarrow 0, \quad \text { as } t_{2}-t_{1} \rightarrow 0 .
$$

Therefore, (15) and (16) imply that $F u$ is equicontinuous for all $u \in \bar{P}_{m}$. By applying the Arzela-Ascoli theorem on time scales, we can see that $F \bar{P}_{m}$ is relatively compact. In view of Lebesgue's dominated convergence theorem on time scales, it is clear that $F$ is a continuous operator. Hence, $F: P \rightarrow P$ is completely continuous operator. The proof is complete. 


\section{Main results}

For $u \in P$, we define

$$
\begin{aligned}
& \alpha(u)=\max _{t \in[0,1]_{\mathbb{T}}}|u(t)|=u(1), \quad \beta(u)=\max _{t \in[0,1]_{\mathbb{T}}}\left|u^{\Delta}(t)\right|=u^{\Delta}(0), \\
& \psi(u)=\min _{t \in[\omega, 1]_{\mathbb{T}}} u(t)=u(\omega) .
\end{aligned}
$$

It is easy to see that $\alpha, \beta: P \rightarrow[0, \infty)$ are nonnegative continuous convex functionals with $\|u\|=\max \{\alpha(u), \beta(u)\} ; \psi: P \rightarrow[0, \infty)$ is nonnegative concave functional. We have $\psi(u) \leq$ $\alpha(u)$ for $u \in P$, this means that assumptions $\left(\mathrm{A}_{1}\right)-\left(\mathrm{A}_{3}\right)$ in Lemma 2.1 hold.

Suppose that $\omega \in \mathbb{T}$ with $0<\omega<1$. For convenience, we introduce the following notations:

$$
\begin{aligned}
\Omega:= & \int_{0}^{\omega} G(\omega, s) \phi^{-1}\left(\int_{\omega}^{1} q(\tau) \Delta \tau\right) \Delta s, \\
\Gamma(s):= & \int_{0}^{1} G(s, \gamma) \phi^{-1}\left(\int_{0}^{1} q(\tau) \Delta \tau\right) \Delta \gamma \\
\Lambda:= & \int_{0}^{1} \frac{a}{\rho}(d+c) \phi^{-1}\left(\int_{0}^{1} q(\tau) \Delta \tau\right) \Delta s \\
& +a\left[\rho-\int_{0}^{1} g_{2}(s)(b+a s) \Delta s\right]^{-1} \int_{0}^{1} g_{2}(s)\left(\Gamma(s)+\frac{1}{\rho}(d+c(1-s))\right) \Delta s, \\
E:= & \int_{0}^{1} \frac{d}{\rho}(a+b) \phi^{-1}\left(\int_{0}^{1} q(\tau) \Delta \tau\right) \Delta s+\frac{d}{\rho} \\
& +(b+a)\left[\rho-\int_{0}^{1} g_{2}(s)(b+a s) \Delta s\right]^{-1} \int_{0}^{1} g_{2}(s)\left(\Gamma(s)+\frac{1}{\rho}(d+c(1-s))\right) \Delta s .
\end{aligned}
$$

Theorem 3.1 Assume that $\left(\mathrm{C}_{1}\right)-\left(\mathrm{C}_{4}\right)$ hold. If there exist constants $r_{1}, r, r_{2}, l_{1}$, and $l_{2}$ with $0<r_{1}<r<\frac{r}{\omega} \leq r_{2}, 0<l_{1} \leq l_{2}$ such that $\frac{r}{\Omega} \leq \min \left\{\frac{r_{2}}{E}, \frac{l_{2}}{\Lambda}\right\}$. Suppose further that $f, g_{1}$ satisfy the following three conditions:

(i) $f(t, u, v) \leq \min \left\{\phi\left(\frac{r_{2}}{E}\right), \phi\left(\frac{l_{2}}{\Lambda}\right)\right\}, \int_{0}^{1} g_{1}(s, u) \Delta s \leq \min \left\{\frac{r_{2}}{E}, \frac{l_{2}}{\Lambda}\right\}$, for $(t, u, v) \in[0,1]_{\mathbb{T}} \times\left[0, r_{2}\right] \times\left[-l_{2}, l_{2}\right]$

(ii) $f(t, u, v)>\phi\left(\frac{r}{\Omega}\right)$ for $(t, u, v) \in[\omega, 1]_{\mathbb{T}} \times\left[r, \frac{r}{\omega}\right] \times\left[-l_{2}, l_{2}\right]$;

(iii) $f(t, u, v)<\min \left\{\phi\left(\frac{r_{1}}{E}\right), \phi\left(\frac{l_{1}}{\Lambda}\right)\right\}, \int_{0}^{1} g_{1}(s, u) \Delta s \leq \min \left\{\frac{r_{1}}{E}, \frac{l_{1}}{\Lambda}\right\}$, for $(t, u, v) \in[0,1]_{\mathbb{T}} \times\left[0, r_{1}\right] \times\left[-l_{1}, l_{1}\right]$.

Then problem (5) has at least three nonnegative solutions $u_{1}, u_{2}, u_{3}$, which satisfy

$$
\begin{aligned}
& \max _{t \in[0,1]_{\mathbb{T}}}\left\{u_{1}(t)\right\}<r_{1}, \quad \max _{t \in[0,1]_{\mathbb{T}}}\left|u_{1}^{\Delta}(t)\right|<l_{1} ; \\
& r<\min _{t \in[\omega, 1]_{\mathbb{T}}}\left\{u_{2}(t)\right\} \leq \max _{t \in[0,1]_{\mathbb{T}}}\left\{u_{2}(t)\right\} \leq r_{2}, \quad \max _{t \in[0,1]_{\mathbb{T}}}\left|u_{2}^{\Delta}(t)\right| \leq l_{2} ; \\
& \min _{t \in[\omega, 1]_{\mathbb{T}}}\left\{u_{3}(t)\right\}<r, \quad r_{1}<\max _{t \in[0,1]_{\mathbb{T}}}\left\{u_{3}(t)\right\}<\frac{r}{\omega}, \quad l_{1}<\max _{t \in[0,1]_{\mathbb{T}}}\left|u_{3}^{\Delta}(t)\right| \leq l_{2} .
\end{aligned}
$$

Proof The boundary value problem (5) has a solution $u=u(t)$ if and only if $u$ solves the operator equation $F u=u$. Thus, we set out to verify that the operator $F$ satisfies the generalization of the Leggett-Williams fixed point theorem, which will prove the existence of a fixed point of $F$. 
We first prove that if assumption (i) is satisfied, then $F: \bar{P}\left(\alpha, r_{2} ; \beta, l_{2}\right) \rightarrow \bar{P}\left(\alpha, r_{2} ; \beta, l_{2}\right)$. Let $u \in \bar{P}\left(\alpha, r_{2} ; \beta, l_{2}\right)$, then

$$
\alpha(u)=\max _{t \in[0,1]_{\mathbb{T}}}|u(t)| \leq r_{2}, \quad \beta(u)=\max _{t \in[0,1]_{\mathbb{T}}}\left|u^{\Delta}(t)\right| \leq l_{2},
$$

and assumption (i) implies

$$
f\left(t, u(t), u^{\Delta}(t)\right) \leq \min \left\{\phi\left(\frac{r_{2}}{E}\right), \phi\left(\frac{l_{2}}{\Lambda}\right)\right\}, \quad t \in[0,1]_{\mathbb{T}} .
$$

For all $u \in P$, we have $F u \in P$, therefore,

$$
\begin{aligned}
\alpha(F u)= & \max _{t \in[0,1]_{\mathbb{T}}}|(F u)(t)|=(F u)(1) \\
= & \int_{0}^{1} \frac{d}{\rho}(b+a \sigma(s)) \phi^{-1}\left(\int_{s}^{1} q(\tau) f\left(\tau, u(\tau), u^{\Delta}(\tau)\right) \Delta \tau\right) \Delta s+d \Phi_{f}+(b+a) \Psi_{f} \\
\leq & \int_{0}^{1} \frac{d}{\rho}(b+a \sigma(s)) \phi^{-1}\left(\int_{0}^{1} q(\tau) f\left(\tau, u(\tau), u^{\Delta}(\tau)\right) \Delta \tau\right) \Delta s+d \Phi_{f}+(b+a) \Psi_{f} \\
\leq & \int_{0}^{1} \frac{d}{\rho}(b+a) \phi^{-1}\left(\int_{0}^{1} q(\tau) \phi\left(\frac{r_{2}}{E}\right) \Delta \tau\right) \Delta s+\frac{d r_{2}}{E \rho} \\
& +(a+b) \frac{r_{2}}{E}\left[\rho-\int_{0}^{1} g_{2}(s)(b+a s) \Delta s\right]^{-1} \int_{0}^{1} g_{2}(s)\left(\Gamma(s)+\frac{1}{\rho}(d+c(1-s))\right) \Delta s \\
= & \frac{r_{2}}{E}\left(\int_{0}^{1} \frac{d}{\rho}(b+a) \phi^{-1}\left(\int_{0}^{1} q(\tau) \Delta \tau\right) \Delta s+\frac{d}{\rho}\right. \\
& \left.+(a+b)\left[\rho-\int_{0}^{1} g_{2}(s)(b+a s) \Delta s\right]^{-1} \int_{0}^{1} g_{2}(s)\left(\Gamma(s)+\frac{1}{\rho}(d+c(1-s))\right) \Delta s\right) \\
= & r_{2}
\end{aligned}
$$

and

$$
\begin{aligned}
\beta(F u)= & \max _{t \in[0,1]_{\mathbb{T}}}\left|(F u)^{\Delta}(t)\right|=(F u)^{\Delta}(0) \\
= & \int_{0}^{1} \frac{a}{\rho}(d+c(1-\sigma(s))) \phi^{-1}\left(\int_{s}^{1} q(\tau) f\left(\tau, u(\tau), u^{\Delta}(\tau)\right) \Delta \tau\right) \Delta s-c \Phi_{f}+a \Psi_{f} \\
\leq & \int_{0}^{1} \frac{a}{\rho}(d+c(1-\sigma(s))) \phi^{-1}\left(\int_{s}^{1} q(\tau) f\left(\tau, u(\tau), u^{\Delta}(\tau)\right) \Delta \tau\right) \Delta s+a \Psi_{f} \\
\leq & \int_{0}^{1} \frac{a}{\rho}(d+c(1-\sigma(s))) \phi^{-1}\left(\int_{0}^{1} q(\tau) \phi\left(\frac{l_{2}}{\Lambda}\right) \Delta \tau\right) \Delta s \\
& +\frac{a l_{2}}{\Lambda}\left[\rho-\int_{0}^{1} g_{2}(s)(b+a s) \Delta s\right]^{-1} \int_{0}^{1} g_{2}(s)\left(\Gamma(s)+\frac{1}{\rho}(d+c(1-s))\right) \Delta s \\
= & \frac{l_{2}}{\Lambda}\left(\int_{0}^{1} \frac{a}{\rho}(d+c(1-\sigma(s))) \phi^{-1}\left(\int_{0}^{1} q(\tau) \Delta \tau\right) \Delta s\right. \\
& \left.+a\left[\rho-\int_{0}^{1} g_{2}(s)(b+a s) \Delta s\right]^{-1} \int_{0}^{1} g_{2}(s)\left(\Gamma(s)+\frac{1}{\rho}(d+c(1-s))\right) \Delta s\right) \\
= & l_{2} .
\end{aligned}
$$

Thus, $F \in \bar{P}\left(\alpha, r_{2} ; \beta, l_{2}\right)$ and $F\left(\bar{P}\left(\alpha, r_{2} ; \beta, l_{2}\right)\right) \subset \bar{P}\left(\alpha, r_{2} ; \beta, l_{2}\right)$. 
Secondly, we show that condition $\left(\mathrm{B}_{1}\right)$ of Lemma 2.1 holds. We let $u(t)=\frac{r}{\omega}$ for $t \in[0,1]_{\mathbb{T}}$. It is obvious that $u(t)=\frac{r}{\omega} \in \bar{P}\left(\alpha, \frac{r}{\omega} ; \beta, l_{1}\right)$ and $\psi(u)=\frac{r}{\omega}>r$, and consequently

$$
\left\{u \in \bar{P}\left(\alpha, \frac{r}{\omega} ; \beta, l_{2} ; \psi, r\right): \psi(u)>r\right\} \neq \emptyset .
$$

For all $u \in \bar{P}\left(\alpha, \frac{r}{\omega} ; \beta, l_{2} ; \psi, r\right)$, we have $r \leq u(t) \leq \frac{r}{\omega},\left|u^{\Delta}(t)\right| \leq l_{2}$ for $t \in[\omega, 1]_{\mathbb{T}}$. Thus, by assumption (ii) we get

$$
f\left(t, u(t), u^{\Delta}(t)\right)>\phi\left(\frac{r}{\Omega}\right), \quad \text { for } t \in[\omega, 1]_{\mathbb{T}} .
$$

From the definition of the functional $\psi$ we see that

$$
\begin{aligned}
\psi(F u)= & \min _{t \in[\omega, 1]]_{\mathbb{T}}}|(F u)(t)|=(F u)(\omega) \\
= & \int_{0}^{1} G(\omega, s) \phi^{-1}\left(\int_{s}^{1} q(\tau) f\left(\tau, u(\tau), u^{\Delta}(\tau)\right) \Delta \tau\right) \Delta s \\
& +(d+c(1-\omega)) \Phi_{f}+(b+a \omega) \Psi_{f} \\
\geq & \int_{0}^{1} G(\omega, s) \phi^{-1}\left(\int_{s}^{1} q(\tau) f\left(\tau, u(\tau), u^{\Delta}(\tau)\right) \Delta \tau\right) \Delta s \\
\geq & \int_{0}^{\omega} G(\omega, s) \phi^{-1}\left(\int_{\omega}^{1} q(\tau) f\left(\tau, u(\tau), u^{\Delta}(\tau)\right) \Delta \tau\right) \Delta s \\
> & \int_{0}^{\omega} G(\omega, s) \phi^{-1}\left(\int_{\omega}^{1} q(\tau) \phi\left(\frac{r}{\Omega}\right) \Delta \tau\right) \Delta s \\
= & \frac{r}{\Omega} \int_{0}^{\omega} G(\omega, s) \phi^{-1}\left(\int_{\omega}^{1} q(\tau) \Delta \tau\right) \Delta s=r .
\end{aligned}
$$

So, we obtain $\psi(F u)>r$ for $u \in \bar{P}\left(\alpha, \frac{r}{\omega} ; \beta, l_{2} ; \psi, r\right)$. Therefore, condition $\left(\mathrm{B}_{1}\right)$ of Lemma 2.1 is satisfied.

Thirdly, we show that the condition $\left(\mathrm{B}_{2}\right)$ of Lemma 2.1 is satisfied. For all $u \in \bar{P}\left(\alpha, r_{1} ; \beta, l_{1}\right)$, we have $0 \leq u(t) \leq r_{1},-l_{1} \leq u^{\Delta}(t) \leq l_{1}$ for $t \in[0,1]_{\mathbb{T}}$. From assumption (iii) we obtain

$$
f\left(t, u(t), u^{\Delta}(t)\right)<\min \left\{\phi\left(\frac{r_{1}}{E}\right), \phi\left(\frac{l_{1}}{\Lambda}\right)\right\}, \quad t \in[0,1]_{\mathbb{T}}
$$

Thus

$$
\begin{aligned}
\alpha(F u) & =\max _{t \in[0,1]_{\mathbb{T}}}|(F u)(t)|=(F u)(1) \\
& =\int_{0}^{1} \frac{d}{\rho}(b+a \sigma(s)) \phi^{-1}\left(\int_{s}^{1} q(\tau) f\left(\tau, u(\tau), u^{\Delta}(\tau)\right) \Delta \tau\right) \Delta s+d \Phi_{f}+(b+a) \Psi_{f} \\
& \leq \int_{0}^{1} \frac{d}{\rho}(b+a \sigma(s)) \phi^{-1}\left(\int_{0}^{1} q(\tau) f\left(\tau, u(\tau), u^{\Delta}(\tau)\right) \Delta \tau\right) \Delta s+d \Phi_{f}+(b+a) \Psi_{f} \\
& <\int_{0}^{1} \frac{d}{\rho}(b+a \sigma(s)) \phi^{-1}\left(\int_{0}^{1} q(\tau) \phi\left(\frac{r_{1}}{E}\right) \Delta \tau\right) \Delta s+\frac{d r_{1}}{\rho E}
\end{aligned}
$$




$$
\begin{aligned}
& +(b+a) \frac{r_{1}}{E}\left[\rho-\int_{0}^{1} g_{2}(s)(b+a s) \Delta s\right]^{-1} \int_{0}^{1} g_{2}(s)\left(\Gamma(s)+\frac{1}{\rho}(d+c(1-s))\right) \Delta s \\
= & \frac{r_{1}}{E}\left(\int_{0}^{1} \frac{d}{\rho}(a+b) \phi^{-1}\left(\int_{0}^{1} q(\tau) \Delta \tau\right) \Delta s+\frac{d}{\rho}\right. \\
& \left.+(b+a)\left[\rho-\int_{0}^{1} g_{2}(s)(b+a s) \Delta s\right]^{-1} \int_{0}^{1} g_{2}(s)\left(\Gamma(s)+\frac{1}{\rho}(d+c(1-s))\right) \Delta s\right) \\
= & r_{1}
\end{aligned}
$$

and

$$
\begin{aligned}
\beta(F u)= & \max _{t \in[0,1]_{\mathbb{T}}}\left|(F u)^{\Delta}(t)\right|=(F u)^{\Delta}(0) \\
= & \int_{0}^{1} \frac{a}{\rho}(d+c(1-\sigma(s))) \phi^{-1}\left(\int_{s}^{1} q(\tau) f\left(\tau, u(\tau), u^{\Delta}(\tau)\right) \Delta \tau\right) \Delta s-c \Phi_{f}+a \Psi_{f} \\
\leq & \int_{0}^{1} \frac{a}{\rho}(d+c(1-\sigma(s))) \phi^{-1}\left(\int_{s}^{1} q(\tau) f\left(\tau, u(\tau), u^{\Delta}(\tau)\right) \Delta \tau\right) \Delta s+a \Psi_{f} \\
< & \int_{0}^{1} \frac{a}{\rho}(d+c) \phi^{-1}\left(\int_{0}^{1} q(\tau) \phi\left(\frac{l_{1}}{\Lambda}\right) \Delta \tau\right) \Delta s \\
& +\frac{a l_{1}}{\Lambda}\left[\rho-\int_{0}^{1} g_{2}(s)(b+a s) \Delta s\right]^{-1} \int_{0}^{1} g_{2}(s)\left(\Gamma(s)+\frac{1}{\rho}(d+c(1-s))\right) \Delta s \\
= & \frac{l_{1}}{\Lambda}\left(\int_{0}^{1} \frac{a}{\rho}(d+c) \phi^{-1}\left(\int_{0}^{1} q(\tau) \Delta \tau\right) \Delta s\right. \\
& \left.+a\left[\rho-\int_{0}^{1} g_{2}(s)(b+a s) \Delta s\right]^{-1} \int_{0}^{1} g_{2}(s)\left(\Gamma(s)+\frac{1}{\rho}(d+c(1-s))\right) \Delta s\right) \\
= & l_{1} .
\end{aligned}
$$

We get $F: \bar{P}\left(\alpha, r_{1} ; \beta, l_{1}\right) \rightarrow P\left(\alpha, r_{1} ; \beta, l_{1}\right)$, which means that $\left(\mathrm{B}_{2}\right)$ in Lemma 2.1 is satisfied.

Finally, we show that

$$
\psi(F u)>b \quad \text { for } u \in \bar{P}\left(\alpha, r_{2} ; \beta, l_{2} ; \psi, b\right) \text { with } \alpha(F u)>d \text {. }
$$

It is easy to see that $(F u)^{\Delta \Delta}(t) \leq 0$ for any $t \in[0,1]_{\mathbb{T}}$. Hence $(F u)^{\Delta}$ is a decreasing function on $[0,1]_{\mathbb{T}}$. This means that graph of $F u$ is concave down on $(\omega, 1)_{\mathbb{T}}$. So, we have

$$
\frac{(F u)(\omega)-(F u)(0)}{\omega} \geq(F u)(1)-(F u)(0)
$$

namely,

$$
(F u)(\omega) \geq \omega(F u)(1)+(1-\omega)(F u)(0) \geq \omega(F u)(1) .
$$

For all $u \in \bar{P}\left(\alpha, r_{2} ; \beta, l_{2} ; \psi, r\right)$ with $\alpha(F u)>\frac{r}{\omega}$ and (17), we have

$$
\psi(F u)=\min _{t \in[\omega, 1]_{\mathbb{T}}} F u(t)=F u(\omega) \geq \omega F u(1)=\omega \max _{t \in[0,1]_{\mathbb{T}}} F u(t)=\omega \alpha(F u)>r .
$$


Therefore, condition $\left(\mathrm{B}_{3}\right)$ of Lemma 2.1 is satisfied. So, all the conditions of Lemma 2.1 are satisfied. It follows from Lemma 2.1 and the assumption that $f(t, 0,0) \neq 0$ on $[0,1]_{\mathbb{T}}$ that $F$ has at least three fixed points $u_{1}, u_{2}, u_{3}$ satisfying

$$
\begin{aligned}
& \max _{t \in[0,1]_{\mathbb{T}}}\left\{u_{1}(t)\right\}<r_{1}, \quad \max _{t \in[0,1]_{\mathbb{T}}}\left|u_{1}^{\Delta}(t)\right|<l_{1} ; \\
& r<\min _{t \in[\omega, 1]_{\mathbb{T}}}\left\{u_{2}(t)\right\} \leq \max _{t \in[0,1]_{\mathbb{T}}}\left\{u_{2}(t)\right\} \leq r_{2}, \quad \max _{t \in[0,1]_{\mathbb{T}}}\left|u_{2}^{\Delta}(t)\right| \leq l_{2} ; \\
& \min _{t \in[\omega, 1]_{\mathbb{T}}}\left\{u_{3}(t)\right\}<r, \quad r_{1}<\max _{t \in[0,1]_{\mathbb{T}}}\left\{u_{3}(t)\right\}<\frac{r}{\omega}, \quad l_{1}<\max _{t \in[0,1]_{\mathbb{T}}}\left|u_{3}^{\Delta}(t)\right| \leq l_{2} .
\end{aligned}
$$

The proof is complete.

If $\phi(x)=\Phi_{p}(x)=|x|^{p-2} x$ for some $p>1$, where $\Phi_{p}^{-1}=\Phi_{q}$, then (5) can be written as a BVP with a $p$-Laplace operator:

$$
\left\{\begin{array}{l}
\left(\Phi_{p}\left(-u^{\Delta \Delta}(t)\right)\right)^{\Delta}+q(t) f\left(t, u(t), u^{\Delta}(t)\right)=0, \quad t \in[0,1]_{\mathbb{T}} \\
a u(0)-b u^{\Delta}(0)=\int_{0}^{1} g_{1}(s, u(s)) \Delta s, \\
c u(1)+d u^{\Delta}(1)=\int_{0}^{1} g_{2}(s) u(s) \Delta s \\
u^{\Delta \Delta}(1)=0 .
\end{array}\right.
$$

Then, by Theorem 3.1, we have the following.

Corollary 3.1 Assume that $\left(\mathrm{C}_{1}\right)-\left(\mathrm{C}_{5}\right)$ hold. If there exist constants $r_{1}, r, r_{2}, l_{1}$, and $l_{2}$ with $0<r_{1}<r<\frac{r}{\omega} \leq r_{2}, 0<l_{1} \leq l_{2}$ such that $\frac{r}{\Omega} \leq \min \left\{\frac{r_{2}}{E}, \frac{l_{2}}{\Lambda}\right\}$. Suppose further that $f, g_{1}$ satisfy the following three conditions:

(i) $f(t, u, v) \leq \min \left\{\Phi_{p}\left(\frac{r_{2}}{E}\right), \Phi_{p}\left(\frac{l_{2}}{\Lambda}\right)\right\}, \int_{0}^{1} g_{1}(s, u) \Delta s \leq \min \left\{\frac{r_{2}}{E}, \frac{l_{2}}{\Lambda}\right\}$, for

$$
(t, u, v) \in[0,1]_{\mathbb{T}} \times\left[0, r_{2}\right] \times\left[-l_{2}, l_{2}\right]
$$

(ii) $f(t, u, v)>\Phi_{p}\left(\frac{r}{\Omega}\right)$ for $(t, u, v) \in[\omega, 1]_{\mathbb{T}} \times\left[r, \frac{r}{\omega}\right] \times\left[-l_{2}, l_{2}\right]$;

(iii) $f(t, u, v)<\min \left\{\Phi_{p}\left(\frac{r_{1}}{E}\right), \Phi_{p}\left(\frac{l_{1}}{\Lambda}\right)\right\}, \int_{0}^{1} g_{1}(s, u) \Delta s \leq \min \left\{\frac{r_{1}}{E}, \frac{l_{1}}{\Lambda}\right\}$, for $(t, u, v) \in[0,1]_{\mathbb{T}} \times\left[0, r_{1}\right] \times\left[-l_{1}, l_{1}\right]$.

Then problem (18) has at least three nonnegative solutions $u_{1}, u_{2}, u_{3}$, which satisfy

$$
\begin{aligned}
& \max _{t \in[0,1]_{\mathbb{T}}}\left\{u_{1}(t)\right\}<r_{1}, \quad \max _{t \in[0,1]_{\mathbb{T}}}\left|u_{1}^{\Delta}(t)\right|<l_{1} ; \\
& r<\min _{t \in[\omega, 1]_{\mathbb{T}}}\left\{u_{2}(t)\right\} \leq \max _{t \in[0,1]_{\mathbb{T}}}\left\{u_{2}(t)\right\} \leq r_{2}, \quad \max _{t \in[0,1]_{\mathbb{T}}}\left|u_{2}^{\Delta}(t)\right| \leq l_{2} ; \\
& \min _{t \in[\omega, 1]_{\mathbb{T}}}\left\{u_{3}(t)\right\}<r, \quad r_{1}<\max _{t \in[0,1]_{\mathbb{T}}}\left\{u_{3}(t)\right\}<\frac{r}{\omega}, \quad l_{1}<\max _{t \in[0,1]_{\mathbb{T}}}\left|u_{3}^{\Delta}(t)\right| \leq l_{2} .
\end{aligned}
$$

\section{An example}

Let $\mathbb{T}=[0,1]$. Take $q(t)=1, a=3, b=1, c=\frac{1}{6}$, and $d=4$ for $t \in[0,1]$. Consider the following BVP:

$$
\left\{\begin{array}{l}
\left(\phi\left(-u^{\Delta \Delta}(t)\right)\right)^{\Delta}+f\left(t, u(t), u^{\Delta}(t)\right)=0, \quad t \in[0,1] \\
3 u(0)-u^{\Delta}(0)=\int_{0}^{1} g_{1}(s, u(s)) \Delta s \\
\frac{1}{6} u(1)+4 u^{\Delta}(1)=\int_{0}^{1} g_{2}(s) u(s) \Delta s \\
u^{\Delta \Delta}(1)=0
\end{array}\right.
$$


where

$$
f(t, u, v)= \begin{cases}\frac{t}{50}+\frac{2}{3} u^{3}+\left(\frac{v}{100}\right)^{3}, & u \leq 3 \\ \frac{t}{50}+\frac{54}{3}+\left(\frac{v}{100}\right)^{3}, & u>3\end{cases}
$$

and

$$
\phi(u)=u, \quad g_{1}(t, u)=t^{2} u+\left(\frac{u}{100}\right)^{3} t, \quad g_{2}(t)=1 .
$$

By calculating, we have $\rho=\frac{38}{3}$, and

$$
G(t, s)=\frac{3}{38} \begin{cases}(1+3 s)\left(\frac{25}{6}-\frac{t}{6}\right), & 0 \leq s \leq t \leq 1 \\ (1+3 t)\left(\frac{25}{6}-\frac{s}{6}\right), & 0 \leq t \leq s \leq 1 .\end{cases}
$$

Set $\omega=\frac{1}{2}$, then we obtain

$$
\Omega=\frac{207}{608}, \quad \Lambda=\frac{11,781}{9,272}, \quad E=\frac{4,537}{2,318} .
$$

Clearly, assumptions $\left(\mathrm{C}_{1}\right)-\left(\mathrm{C}_{5}\right)$ hold and $f(t, 0,0) \neq 0$ on $[0,1]$. We choose $r_{1}=\frac{1}{2}, r=3$, $r_{2}=120$, and $l_{1}=\frac{1}{4}, l_{2}=90$. So $0<r_{1}<r<\frac{r}{\omega}$ and $0<l_{1}<l_{2}$, and it is easy to check that $\frac{r}{\Omega} \leq \min \left\{\frac{r_{2}}{E}, \frac{l_{2}}{\Lambda}\right\}$. Now, we show that conditions (i)-(iii) are satisfied:

(i) $f(t, u, v) \leq 18.020<61.309 \approx \min \left\{\phi\left(\frac{r_{2}}{E}\right), \phi\left(\frac{l_{2}}{\Lambda}\right)\right\}$, $\int_{0}^{1} g_{1}(s, u(s)) \Delta s \leq \int_{0}^{1}\left(120 t^{2}+\left(\frac{120}{100}\right)^{3} t\right) \Delta s=40.864<61.309 \approx \min \left\{\frac{r_{2}}{E}, \frac{l_{2}}{\Lambda}\right\}$, for $(t, u, v) \in[0,1] \times[0,120] \times\left[-\frac{1}{4}, \frac{1}{4}\right]$

(ii) $f(t, u, v) \geq 17.281>8.812 \approx \phi\left(\frac{r}{\Omega}\right)$, for $(t, u, v) \in\left[\frac{1}{2}, 1\right] \times[3,6] \times[-90,90]$;

(iii) $f(t, u, v) \leq 0.1033<0.197 \approx \min \left\{\phi\left(\frac{r_{1}}{E}\right), \phi\left(\frac{l_{1}}{\Lambda}\right)\right\}$,

$$
\begin{aligned}
& \int_{0}^{1} g_{1}(s, u(s)) \Delta s \leq \int_{0}^{1} \frac{1}{2} t^{2} \Delta s \approx 0.1667<0.197 \approx \min \left\{\frac{r_{1}}{E}, \frac{l_{1}}{\Lambda}\right\}, \text { for } \\
& (t, u, v) \in[0,1] \times\left[0, \frac{1}{2}\right] \times\left[-\frac{1}{4}, \frac{1}{4}\right] .
\end{aligned}
$$

From the above, we see that all the conditions of Theorem 3.1 are satisfied. Hence, by Theorem 3.1, BVP (19) has at least three nonnegative solutions $u_{1}, u_{2}, u_{3}$ such that

$$
\begin{aligned}
& \max _{t \in[0,1]_{\mathbb{T}}}\left\{u_{1}(t)\right\}<\frac{1}{2}, \quad \max _{t \in[0,1]_{\mathbb{T}}}\left|u_{1}^{\Delta}(t)\right|<\frac{1}{4} ; \\
& 3<\min _{t \in[0,1]_{\mathbb{T}}}\left\{u_{2}(t)\right\} \leq \max _{t \in[0,1]_{\mathbb{T}}}\left\{u_{2}(t)\right\} \leq 120, \quad \max _{t \in[0,1]_{\mathbb{T}}}\left|u_{2}^{\Delta}(t)\right| \leq 90 ; \\
& \min _{t \in[\omega, 1]_{\mathbb{T}}}\left\{u_{3}(t)\right\}<3, \quad \frac{1}{2}<\max _{t \in[0,1]_{\mathbb{T}}}\left\{u_{3}(t)\right\}<6, \quad \frac{1}{4}<\max _{t \in[0,1]_{\mathbb{T}}}\left|u_{3}^{\Delta}(t)\right| \leq 90 .
\end{aligned}
$$

\section{Competing interests}

The authors declare that they have no competing interests.

\section{Authors' contributions}

All authors wrote, read, and approved the final manuscript.

\section{Acknowledgements}

The authors would like to thank the referees for their valuable comments and suggestions. This study was supported by the National Natural Sciences Foundation of People's Republic of China under Grant 11361072.

Received: 6 February 2015 Accepted: 13 March 2015 Published online: 19 March 2015 


\section{References}

1. Timoshenko, S: Theory of Elastic Stability. McGraw-Hill, New York (1961)

2. Yang, ZL: Positive solutions of a second order integral boundary value problem. J. Math. Anal. Appl. 321, 751-765 (2006)

3. Zhang, XM, Feng, MQ, Ge, WG: Existence of solutions of boundary value problems with integral boundary conditions for second-order impulsive integro-differential equations in Banach spaces. Comput. Appl. Math. 233, 1915-1926 (2010)

4. Feng, MQ: Existence of symmetric positive solutions for a boundary value problem with integral boundary conditions. Appl. Math. Lett. 24, 1419-1427 (2011)

5. Guo, YP, Liu, YJ, Liang, YC: Positive solutions for the third-order boundary value problems with the second derivatives. Bound. Value Probl. 2012, Article ID 34 (2012)

6. Bohner, M, Peterson, A: Dynamic Equations on Time Scales: An Introduction with Applications. Birkhäuser, Boston (2001)

7. Bohner, M, Peterson, A: Advances in Dynamic Equations on Time Scales. Birkhäuser, Boston (2003)

8. Lakshmikantham, V, Sivasundaram, S, Kaymakcalan, B: Dynamic Systems on Measure Chains. Kluwer Academic, Boston (1996)

9. Li, YK, Shu, JY: Multiple positive solutions for first-order impulsive integral boundary value problems on time scales. Bound. Value Probl. 2011, Article ID 12 (2011)

10. Li, YK, Sun, LJ: Infinite many positive solutions for nonlinear first-order BVPs with integral boundary conditions on time scales. Topol. Methods Nonlinear Anal. 41, 305-321 (2013)

11. Agarwal, RP, Otero-Espinar, V, Perera, K, Vivero, DR: Multiple positive solutions of singular Dirichlet problems on time scales via variational methods. Nonlinear Anal. 67, 368-381 (2007)

12. Jiang, LQ, Zhou, Z: Existence of weak solutions of two-point boundary value problems for second-order dynamic equations on time scales. Nonlinear Anal. 69, 1376-1388 (2008)

13. Rynne, BP: $L^{2}$ Spaces and boundary value problems on time-scales. J. Math. Anal. Appl. 328, 1217-1236 (2007)

14. Zhou, JW, Li, YK: Sobolev's spaces on time scales and its applications to a class of second order Hamiltonian systems on time scales. Nonlinear Anal. 73, 1375-1388 (2010)

15. Zhou, JW, Li, YK: Variational approach to a class of second order Hamiltonian systems on time scales. Acta Appl. Math 117, 47-69 (2012)

16. Tokmak, F, Karaca, IY: Existence of positive solutions for third-order boundary value problems with integral boundary conditions on time scales. J. Inequal. Appl. 2013, Article ID 498 (2013)

17. Li, YK, Zhang, TW: Multiple positive solutions for second-order $p$-Laplacian dynamic equations with integral boundary conditions. Bound. Value Probl. 2011, Article ID 867615 (2011)

18. Han, W, Kang, SG: Multiple positive solutions of nonlinear third-order BVP for a class of $p$-Laplacian dynamic equations on time scales. Math. Comput. Model. 49, 527-535 (2009)

19. Bai, ZB, Ge, WG: Existence of three positive solutions for some second-order boundary value problems. Comput. Math. Appl. 48, 699-707 (2004)

20. Deimling, K: Nonlinear Functional Analysis. Springer, New York (1985)

21. Guo, D, Lakshmikantham, V: Nonlinear Problems in Abstract Cones. Academic Press, New York (1988)

\section{Submit your manuscript to a SpringerOpen ${ }^{\circ}$ journal and benefit from:}

- Convenient online submission

- Rigorous peer review

- Immediate publication on acceptance

- Open access: articles freely available online

- High visibility within the field

Retaining the copyright to your article 